\title{
DENSITY AND VELOCITY MEASUREMENTS IN TURBULENT HE-AIR BOUNDARY LAYERS
}

\author{
Received 13/08/2001-Accepted 17/05/2003
}

\begin{abstract}
A turbulent boundary layer with large density variations has been generated by tangential injection of air or helium Into a boundary layer of air-helium mixture. Instrumentation based on thermoanemometry has been successfully developed for the investigation of this flow Analysis or the mean and fluctuating density fields shows that the flow is mainly governed by the ratio of the injection to the external velocity and that the density ratio plays a secondary role in the mixing processes. However, a sight enhancement of turbulent activity is observed when helium is injected.

Keywords: Turbulent boundary layer, mixture of gas, flow with variable density, thermoanemometry.

\section{Résumé}

Une couche limite turbulente avec différences de densité importantes a été produite par injection tangentielle d'air ou d'hélium dans un mélange de ces deux constituants. L'instrumentation, basée sur la thermo-anémométrie, a été développée avec succès pour l'étude de ce type d'écoulement. L'analyse des résultats obtenus, à travers les champs moyen et fluctuant de densité, montre que l'écoulement est principalement régi par le rapport de l'injection à la vitesse externe, et que le rapport de densité joue un rôle secondaire dans les processus de mélange. Cependant, on observe un perfectionnement du point de vue de l'activité turbulente quand l'hélium est injecté.
\end{abstract}

Mots clés: Couche limite turbulente, mélange de gaz, écoulement à densité variable, thermo-anémométrie.

\section{A. SOUDANI \\ S. BOUGOUL}

Département de Physique

Faculté des Sciences

Université de Batna

Batna, Algérie

\section{J.L. HARION}

Laboratoire des Ecoulements Géophysiques et Industriels BP.53X, 38041, Grenoble

Cedex, France

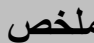

ندرس في هذا المقال طبقة حدية مضطربة ذات

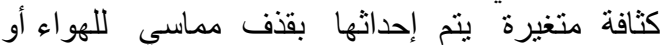

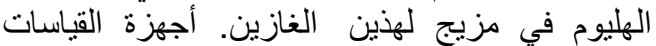

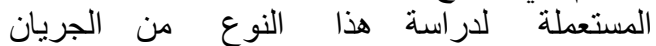

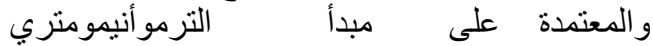

تم تطوير ها بنجاح. تحليل (Thermo-anemometry)

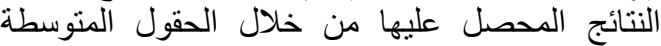

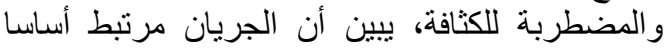
بنسبة القذف إلى السر عة الخارجية وانية ويبين كذللك أن نسبة

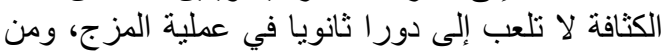

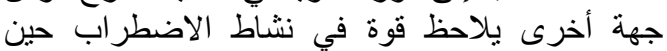

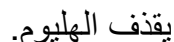

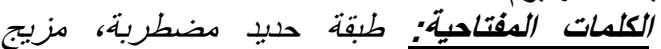

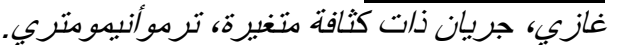

\section{1- AIM OF RESEARCH}

Turbulent boundary layers with large density variation occur in the presence of large temperature differences between the wall and the outer flow as on the space shuttle during re-entry into the atmosphere or the turbine blades cooled by tangential injection. Accurate predictions of heat transfer which are crucial for design purposes raise the question of the influence of differences on turbulent transport properties. Only few studies have been devoted to detailed investigations of turbulent quantities in such flows [1-3] and some results published therein seem, moreover, questionable (e.g. the very high values of normal velocity fluctuation intensity near the wall in a helium/air boundary layer obtained by Larue and Libby). In these investigations, wall quantities such as skin friction and heat transfer coefficients were not measured and there is no information available on the effects of large density variations on the transport properties very near the wall. In particular in computations, the question of wall boundary conditions arises for density fluctuations. In the heat transfer problem, there exists a complex wall-flow interaction and, due to unsteady conduction into the wall, the temperature fluctuations at the wall are not necessarily zero [4].

In the mass transfer case, the condition of impermeable wall implies:

$$
\left(\frac{\partial \bar{\rho}}{\partial y}\right)_{w}=0 \quad\left(\frac{\partial \bar{\rho}^{\prime 2}}{\partial y}\right)_{w}=0
$$

This last condition is compatible with non-zero density fluctuations at the wall.

The aim of the present experimental investigation is to contribute to the knowledge and understanding of effects of large density variations on turbulent wall flows, in particular of possible effects on density fluctuations on the skin friction at the wall. Instrumentation derived from the original work of Way and Libby [5] has been developed for simultaneous measurements of turbulent density/velocity fluctuations in the flow or density/shear stress fluctuations at the wall. 


\section{2- EXPERIMENTAL SET-UP- INSTRUMENTATION}

\section{1- Experimental arrangement}

Density differences in the boundary layer are generated by injecting either air or helium parallel to the wall (Fig.1) in a way similar to the experiment of LaRue and Libby (referenced LR-L hereafter). More precisely, the external flow is tripped boundary layer of air-helium mixtures (velocity $U_{\infty}$, density $\rho_{\infty}$ ) and laminar jet flow (bulk velocity $U_{i n j}$, density $\rho_{i n j}$ ) is injected tangentially through as slot located at $x=577 \mathrm{~mm}$ from the leading edge of the upper plate. The injection slot width $(e=3 \mathrm{~mm})$ and the external density $\left(\rho_{\infty}=1.0 \mathrm{~kg} / \mathrm{m}^{3}\right)$ have been kept constant during the experiments.

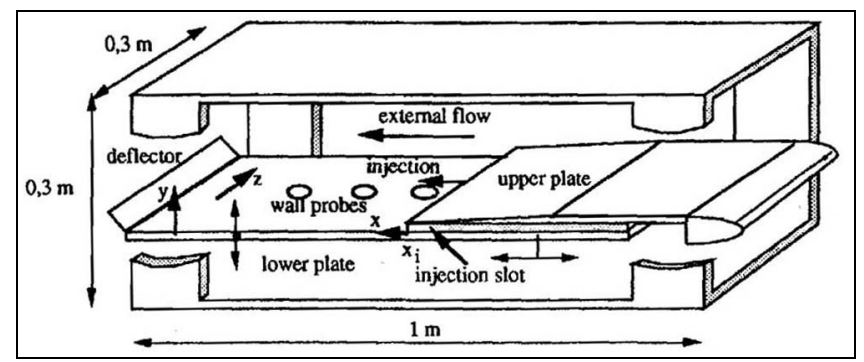

Figure 1: Wind tunnel. Test section equipment.

\section{2- Flow conditions}

The parameters of the flow are:

- The Reynolds number of the injected fluid at the slot exit :

$\operatorname{Re}=U_{i n j} e / v_{i n j}$

- The density ratio $\quad \mathrm{S}=\rho_{i n j} / \rho_{\infty}$

- The velocity ratio $\quad r=U_{i n j} / U_{\infty}$

The mass flow ratio $r_{m}(=S r)$ and the momentum ratio $r_{M}\left(=S r^{2}\right)$ are deduced from the preceding parameters.

In order to investigate the influence of flow conditions on development of the boundary layer, different experiments (table 1) have been performed. Experiments 1 and 2 correspond to a constant $r$ value $: r=0.345$, whereas injection of helium in experiment 3 and injection of air in experiment 4 correspond to the same $r_{M}(0.035)$.

Most measurements have been performed in the conditions of experiment 1 . The boundary layer thickness defined as the value of y corresponding to $\bar{U}=0.99 U_{\infty}$, $\delta(x=577 \mathrm{~mm})$ is $20.5 \mathrm{~mm}$ in this case. Effects of buoyancy are negligible in this experiment: $\left(R i=g|1-S| e / U_{\infty}^{2}<10^{-3}\right)$.

\begin{tabular}{|c|c|c|c|c|c|c|}
\cline { 4 - 7 } \multicolumn{2}{c|}{} & \multicolumn{2}{|c|}{$\begin{array}{c}\text { Injection of He } \\
\boldsymbol{S}=\mathbf{0 . 1 6}\end{array}$} & \multicolumn{2}{|c|}{$\begin{array}{c}\text { Injection of air } \\
\boldsymbol{S}=\mathbf{1 . 2}\end{array}$} \\
\hline \multirow{E}{*}{ Exp. } & $\begin{array}{c}U_{\infty} \\
(\mathrm{m} / \mathrm{s})\end{array}$ & $\begin{array}{c}U_{i n j} \\
(\mathrm{~m} / \mathrm{s})\end{array}$ & $r_{m}$ & $r_{M}$ & $r_{m}$ & $r_{M}$ \\
\hline 1 & 5.8 & 2 & 0.058 & 0.02 & 0.417 & 0.143 \\
\hline 2 & 11.6 & 4 & 0.058 & 0.02 & 0.417 & 0.143 \\
\hline 3 & 5.8 & 2.7 & 0.076 & 0.035 & - & - \\
\hline 4 & 5.8 & 1 & - & - & 0.207 & 0.035 \\
\hline
\end{tabular}

Table 1: Flow conditions.

\section{3- Instrumentation}

Measurements of longitudinal velocity $U$, wall shearstress $\tau$ and density $\rho$ are performed via hot-wire/film anemometry and aspirating probes based on the hot-wire sensitivity to velocity and density. $U$ and $\rho$ are obtained with a hot-film/hot-wire interfering probe similar to that used by LR-L (Fig. 2). It was found that, contrary to the LR-L configuration, the separation of $U$ and $\rho$ in the response of the two sensors over an extended range of flow conditions is much butter when the upstream wire is at higher temperature than the downstream film. Indeed, the thermal power exchanged by convection between the hot wire and the out-flow depends on the temperature that is imposed by the anemometer and the thermal field in which it is emerged. This last is bound directly to the overheating temperature of the film. The extension of the thermal field toward the upstream can be characterized by a typical length $\xi$. In first approximation, this length is determined by a balance between the convection (velocity $U$ ) and the longitudinal heat diffusion in the fluid (thermal diffusity of the mixture $\alpha$ ). One has then:

$$
U \frac{\Delta T}{\xi} \sim \alpha \frac{\Delta T}{\xi^{2}} \Rightarrow \xi \sim \frac{\alpha}{U}
$$

The film, by its proximity, influences therefore the wire response. On the other hand, because of the weak size of the wire and its moderate overheating, this one doesn't affect the response of the film. In homogeneous boundary layer which develops on the plate, the profiles of the longitudinal mean velocity and of its turbulent intensity measured with such a probe are in good agreement with other data [6].

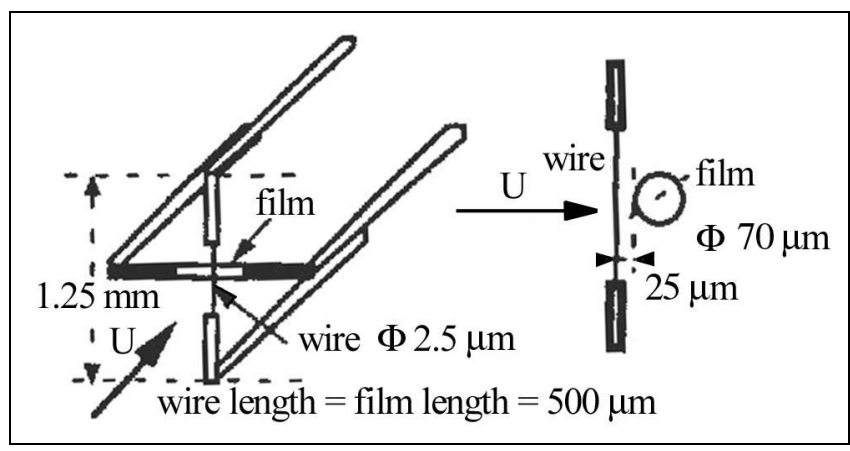

Figure 2: Flow interfering probe.

The density at the wall $\rho_{W}$ is measured directly by an aspirating probe which is insensitive to velocity fluctuations and the skin friction is given by a flush-mounted hot-film located in the same section (Fig. 3).

In the present arrangement, the hot-wire is placed at the entrance of the suction orifice. This improves the timeresponse of the probe (an estimation from concentration spectra indicates a response time less then $10 \mathrm{~ms}$ ). The aspirating probe presents, however, the drawback of integrating the density over a volume of the order of $1 \mathrm{~mm}$. Moreover, the $9 \mathrm{~mm}$ distance between the two sensing elements corresponds to $\Delta \mathrm{z}^{+} \approx 100\left(\Delta z^{+}=\Delta z u_{\tau} / v\right)$ which is comparable to the span wise distance between longitudinal vortices. A new probe was, therefore, developed for the 


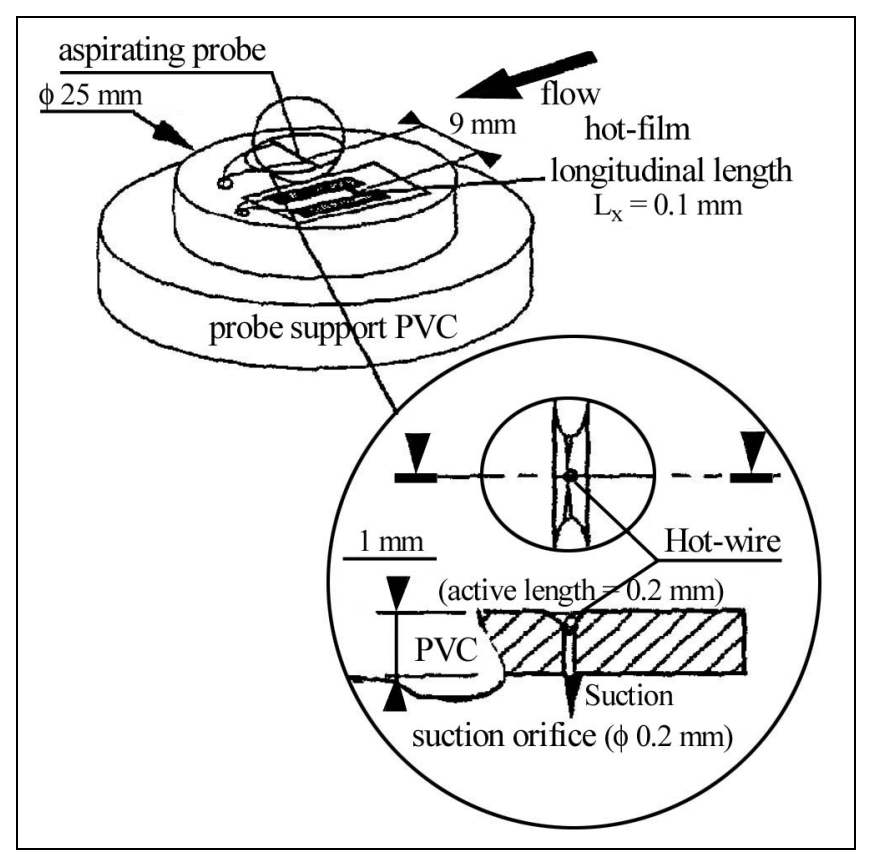

Figure 3: Wall aspirating probe and hot-film arrangement.

simultaneous measurement of density and shear stress fluctuations. It consists of two closely spaced element which interfere thermally (Fig. 4): a flush-mounted hot-film and a hot-wire located in the thermal boundary layer of the upstream film. The response of the flush mounted hot-film must be interpreted with caution because a large fraction of the heat flux from the hot-film goes into the wall by conduction. This effect is taken into account by defining an effective heat transfer length $L$ which is deduced from calibration. All probes are calibrated in laminar channel flow of known velocity and density. The data are processed by using a look-up table and different methods of inversion (interpolation by Spline functions and neural networks ).

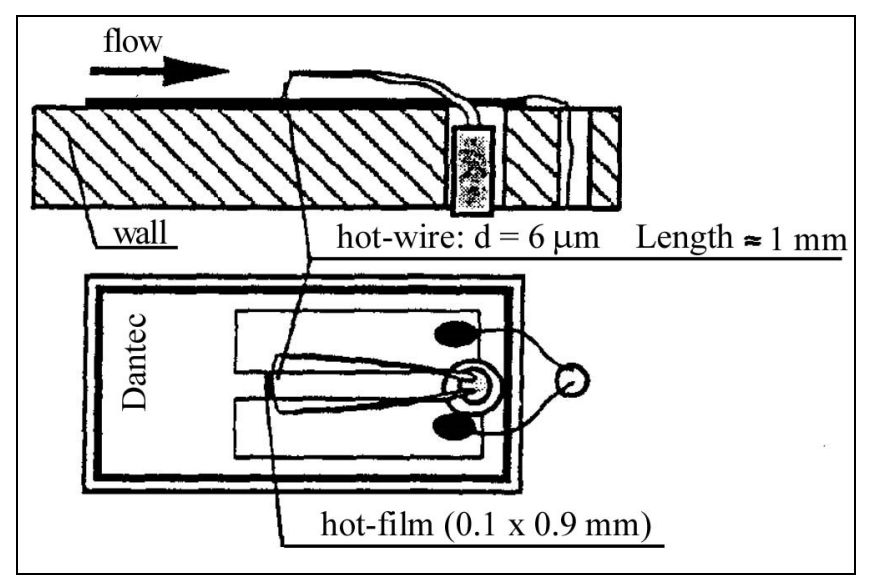

Figure 4: Sketch of the wall interfering probe.

\section{4- Comparison between the different probes}

Figure 5 shows that the different probes used in this investigation give very consistent results for the mean density and the r.m.s density fluctuation along the wall. Data obtained from the wall probes and from movable interference probe placed near the wall (at $y / \delta \approx 0.1$ which corresponds at $x / e=20$ to $y^{+} \approx 15-30$ depending on the injected fluid) have been drawn on the same figures. There is good agreement between the $\bar{\rho}$-measurements of the three probes and the results are consistent with the boundary condition at the wall $(\partial \bar{\rho} / \partial y)_{y=0}=0$. On the contrary, it is expected that presence of the wall has a damping effect on the density fluctuations; indeed the r.m.s given by the movable probe is higher than that of the wall probes. This is particularly true at the beginning of the wall jet $(x / e \approx 10)$ where density fluctuation are very high. It must be noted that the density r.m.s given by the movable probe does not decrease below $2.5 \%$ in the case of air injection. This bias may be due to the rather large timeresponse of the fiber element.
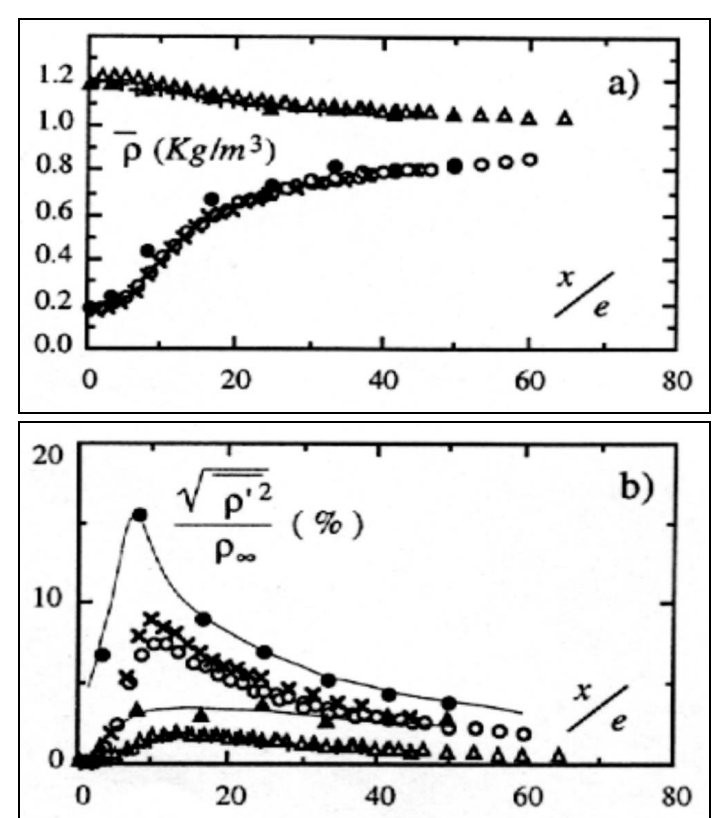

Figure 5: Comparison of the responses of the different probes.

a) Variation of mean density along the wall.

b) Variation of r.m.s of density along the wall.

\begin{tabular}{|l|c|c|c|}
\hline & $\begin{array}{c}\text { Wall interfering } \\
\text { probe }\end{array}$ & $\begin{array}{c}\text { Aspirating } \\
\text { probe }\end{array}$ & $\begin{array}{c}\text { Flow interfering } \\
\text { probe }\end{array}$ \\
\hline Injection of He & $\times$ & $\circ$ & $\bullet$ \\
\hline Injection of air & + & $\Delta$ & $\boldsymbol{\Delta}$ \\
\hline
\end{tabular}

\section{3- RESULTS}

The flow evolves rapidly from an inhomogeneous wall jet-boundary layer combination to a boundary layer with moderate density variation. Detailed results on the mean velocity and density fields are given in Ref. [7-12].

\section{1- Mean velocity and density profiles}

The mean velocity profiles at the initial section result from the superposition of a turbulent boundary layer and of a parabolic channel flow profiles and they evolve toward an asymptotic state. The boundary layer thickness $\delta$ is almost constant along the test section. It is found that the 
$\bar{U}$-profiles are little affected by $S$ at $r$ constant. Injection of helium gives slightly higher velocities near the wall (for $y / \delta<0.4$ ) in the region of developed flow $(x / e>20)$. It follows that the friction velocity $u_{\tau}$ determined by adjustment of the log law to the present $\bar{U}$-profiles is $10 \%$ higher for injection of helium. Profiles drawn with inner variables near the wall for the tow values of $S$.

The development of the wall jet gives rise to a concentration-boundary layer imbedded in the much larger boundary layer which has grown from the leading edge of the upper plat. The mean density has been normalized by the local density difference between the wall and the external flow $\Delta \rho=\left|\rho_{\infty}-\rho_{W}\right|$. Self-similarity of the mean density profiles is observed first in the outer region of the concentration boundary layer; it extends progressively towards the wall in the region of developed flow. The concentration thickness $\delta_{\rho}$, defined as the value of $y$ corresponding to $\left|\rho_{\infty}-\bar{\rho}\right|=0.99 \Delta \rho$, grows rapidly and is given by the following relation for $S=0.16$ : $\delta_{\rho} \approx 1.07+0.41(x / e)^{0.6}$ which is in good agreement with the result of Cheng and $\operatorname{Ng}\left(\approx x^{0.63}\right)$ in a strongly heated boundary layer. $\bar{U}$ and $\bar{\rho}$-profiles at $x / e=34$ are in good agreement with those of LR-L.

\section{2- r.m.s of turbulent velocity and density fluctuations}

Near the wall, the turbulent activity is enhanced by injection of helium. For $y / \delta<0.2$, the velocity fluctuation level increases much more rapidly during the development of the wall jet for $S=0.16$ than for $S=1.2$. Beyond $x / e$ about 20, a small difference between the level of $\sqrt{\overline{u^{12}}} / U_{\infty}$ in the two case of injection is maintained. For $x / e=50$, it may be accented for by the increase of $u_{\tau}$ deduced from the $\bar{U}$-profiles so that $u^{\text {,+ }}$ vs $y^{+}$profiles are in good agreement with those of Purtell et al. in an homogeneous turbulent boundary layer. Unfortunately, due to the geometry of the probe $(1.25 \mathrm{~mm} \times 1.25 \mathrm{~mm})$, it was not possible to reach very small values of $y^{+}$in order to obtain the maximum value of $\sqrt{\overline{u^{\prime 2}}} / U_{\infty}$ and to confirm this result for $y^{+}<20-30$.

The mixing between the two flows at initially different densities gives rise to very high turbulent density fluctuations near the injection slot. Profiles of $\sqrt{\overline{\rho^{\prime 2}}} / \Delta \rho$ present a sharp peak with a maximum value of 0.3 for $x / e=3.3$. These fluctuations amplify further downstream. Figure 6 shows that the level of turbulent density fluctuation is slightly increased by injection of helium. In a strongly heated turbulent boundary layer, Cheng and $\mathrm{Ng}$ found that the $\sqrt{\overline{\rho^{\prime 2}}} / \rho_{\infty}$ profiles reach an asymptotic state with a maximum value of 0.12 .

With the normalization used in the present paper, this corresponds to $\sqrt{\overline{\rho^{\prime 2}}} / \Delta \rho=0.16$, which is much lower that the maximum value observed herein. This may be due to the different boundary condition in the two studies (continuous heating by the wall in Cheng and $\mathrm{Ng}$ study, discontinuous injection of density difference in the present one). Again, the r.m.s profiles of $u^{\prime}$ at $x / e=34$ are in good agreement with those of LR-L. A very strong correlation between density and velocity fluctuations (correlation coefficient $R_{\rho^{\prime} u^{\prime} \text { max }} \approx 0.75$, Fig. 7) is observed when helium is injected.

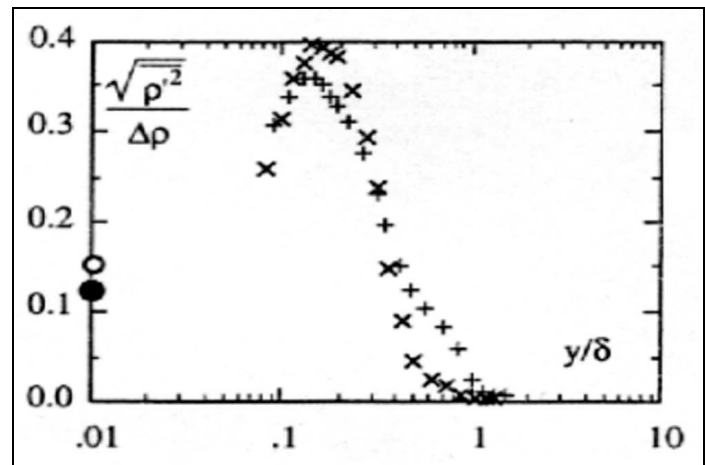

Figure 6: r.m.s of density profiles.

$x / e=16.7-\times:$ injection of helium, + : injection of air. data obtained from the wall measurements, $\circ$ : helium, air.

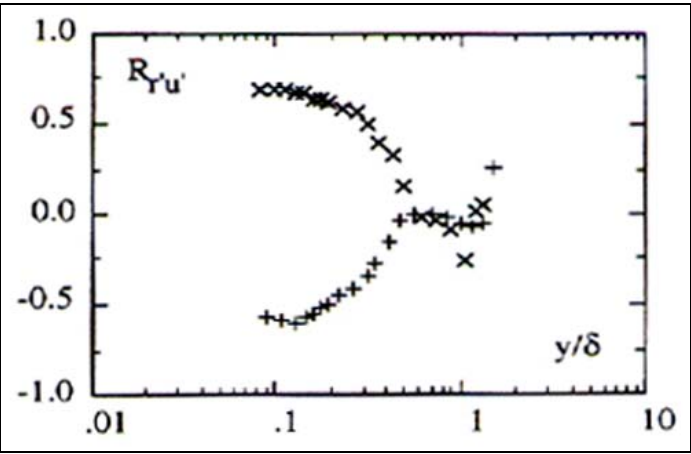

Figure 7: Transverse profiles of velocity / density correlation. Exp. 1: $\mathrm{x} / \mathrm{e}=16.7-\times$ : injection of helium, + : injection of air.

\section{3- Wall measurements}

The following results concerning density have been obtained with the wall aspirating probe.

\subsection{1- Mean density}

The density difference $\Delta \rho$ between the wall and the external flow has been normalized by is initial value: $\eta=\Delta \rho / \Delta \rho_{x=0}$. It can be interpreted as an effectiveness factor $\eta$, referring to the problem of turbine blade cooling. After a transition region, $\eta$ decreases as $(x / e)^{-0.8}$ for all the cases tested (Fig. 8a). this behavior is in agreement with other experiment $[13,14]$. Comparison of experiment 1 and 2 shows that Reynolds number has little effect on the decrease of $\eta$ and that injection of helium gives rise to a slightly faster development of the wall jet when $r$ is kept constant. On the contrary, $\eta$ decreases much faster for 
injection of air when $r_{M}$ is kept constant. It is clear then that the evolution of $\eta$ is governed neither by $r$ nor by $r_{M}$. Following Spalding [15] who reviewed earlier studies on film-cooling systems, the present results collapse well by using a normalized distance to the slot:

$$
X=\left(\frac{x / e}{r}\right)^{0.8} \mathrm{Re}^{-0.2}
$$

On two distinct curves corresponding to the two values of $S$. Collapse on a unique curve (Fig. 8b) is achieved by taking into account a specific effect of $S$ through a correcting factor in relation (1) so that the decrease of the effectiveness is given by:

$$
\eta=4.32\left(\frac{x / e}{r}\right)^{-0.8} \operatorname{Re}^{0.2} S^{-0.1}
$$

Note that the exponent of $S$ is purely empirical.

These results show that the velocity ratio $r$ is the main parameter which governs the mean development of the wall jet. The effects of density are partly taken into account by the viscosity of the injected fluid and partly by the correcting factor $S^{0.1}$.
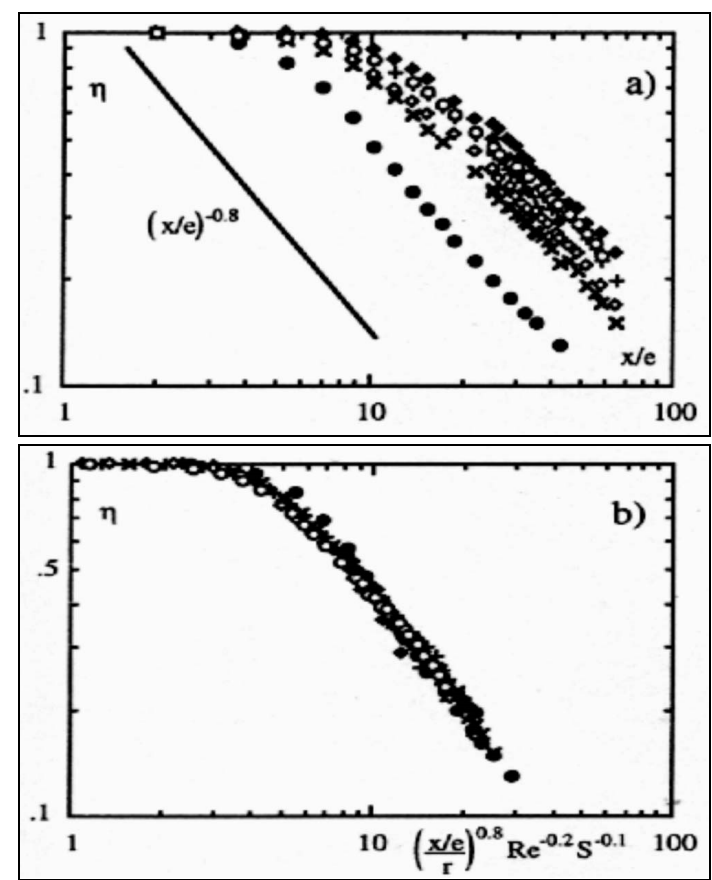

Figure 8: Variations of effectiveness along the wall.

a) normalization by the injection slot width.

b) influence of non-dimensional parameters on effectiveness.

Exp. 1: $\times$ : $S=0.16,+: S=1.2 ;$ Exp. 2: $\circ: S=0.16, \bullet: S=1.2$

Exp. 3: $\bigcirc: S=0.16$; Exp. 4: $: S=1.2$

\subsection{2- R.m.s of the turbulent density fluctuations}

The same conclusion as the mean density can be inferred from the r.m.s of the density fluctuations results at the wall. Figure 9 show that $\left(\sqrt{\overline{\rho^{\prime 2}}}\right)_{w}$ normalized by the local mean density difference $\Delta \rho$ increases_rapidly along the wall and reaches a nearly constant value which is about 0.15 after a transition region of this transition region is slightly shorter for $S=0.16(\approx 17 e)$ than for $S=1.2$ $(\approx 25 e)$. The results do collapse well on a unique curve when the same scaling as for the mean density is used. The tendency to decrease observed for injection of air is questionable since local value of $\Delta \rho$ and $\rho^{\prime}$ are then very small and the inaccuracies in $\left(\sqrt{\overline{\rho^{\prime 2}}}\right)_{w} / \Delta \rho$ become large in the downstream part of the test section. This may also explain the dispersion of the maximum value of $\left(\sqrt{\overline{\rho^{\prime 2}}}\right)_{w} / \Delta \rho$ corresponding to $S=1.2$.

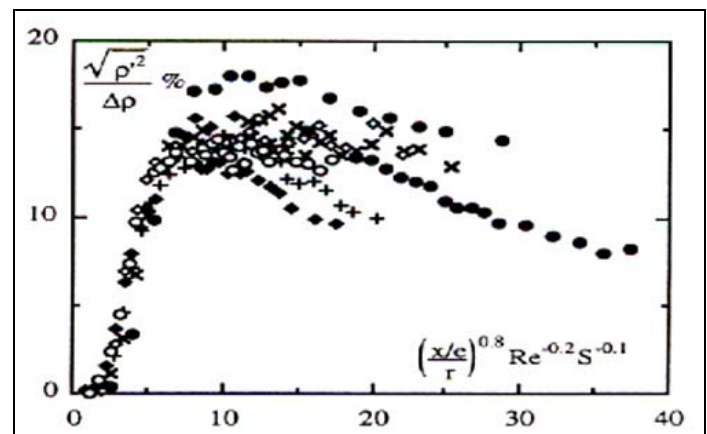

Figure 9: r.m.s of density fluctuations along the wall. Same symbols as in figure 8 .

\subsection{3- Skin friction}

The skin friction coefficient determined directly by the hot-film is approximately constant and independent of the injected gas in the far-field region. The observed value $\left(C_{f}=\tau /\left(0.5 \rho_{\infty} U_{\infty}^{2}\right) \approx 0.004\right.$ in experiment 1) is in $\operatorname{good}$ agreement with other experiment at low Reynolds number [6]. Due to the variation of density, the corresponding friction velocity $u_{\tau}\left(=\left(\tau_{w} / \rho_{w}\right)^{0.5}\right)$ is different for the two cases of injection. Its evolution along the wall (Fig. 10) is consistent with the variation of $u_{\tau}$ as discussed above.

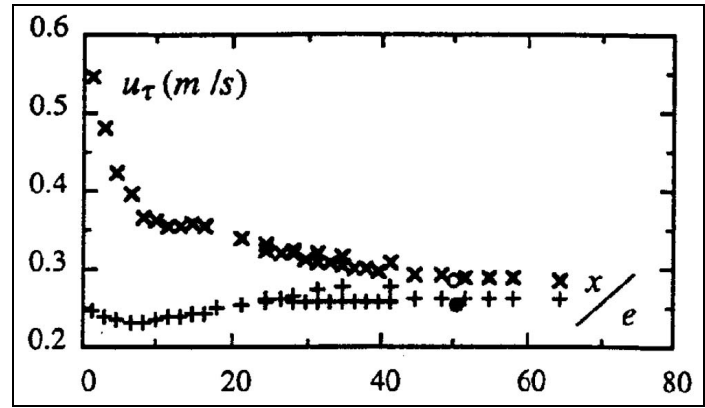

Figure 10: Friction velocity along the wall.

Exp. 1: $\times$ : injection of helium, + : injection of air.

$\bigcirc-$ : Data obtained by adjustment of the mean velocity profiles measured by the interference probe on the log - law of the wall.

The shear stress fluctuations measured by the wall interference probe are tentatively corrected by using the physical length $L_{x}$ instead of the static effective heat transfer length $L$ to interpret the heat flux fluctuations. This 
correction gives a significant increase from $13 \%$ to $38 \%$ of the r.m.s of the measured shear stress for $S=0.16$ in case 1 and brings this result closer to the accepted value in the developed turbulent boundary layer (Fig. 11). For $S=1.2$, however, the corrected value seems less satisfactory $(50 \%)$. Clearly, preliminary results need to be confirmed by further measurements. A better understanding of the physical behavior of the probe in unsteady conditions must be sought to improve accuracy of measurements.
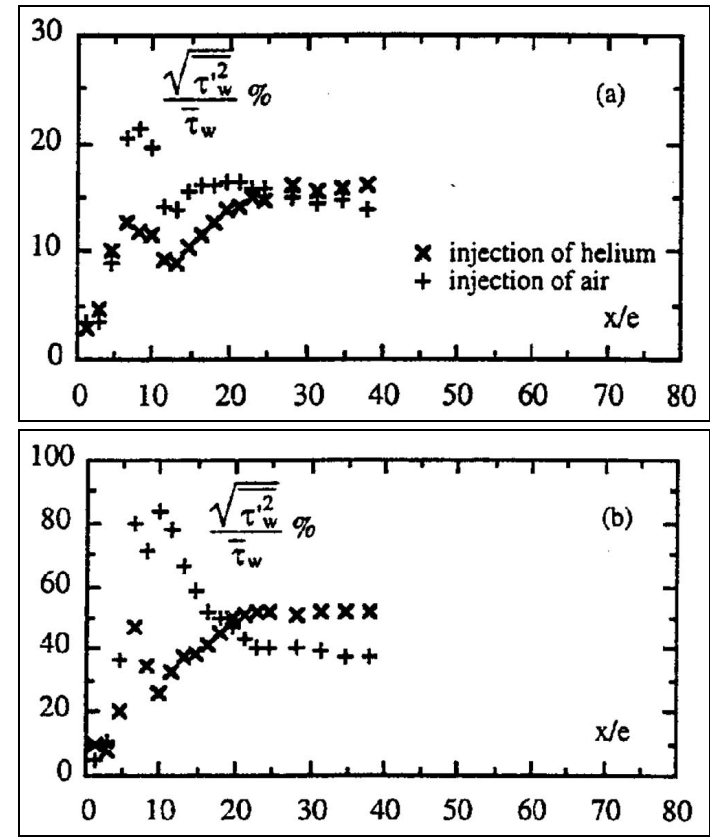

Figure 11: r.m.s of skin friction fluctuations along the wall. a) Results obtained by using the effective heat transfer length $\mathrm{L}$. b) Results obtained by using the physical hot - wire length $L_{x}$.

\section{CONCLUSION}

The present investigation demonstrates the capabilities of new thermo anemometric probes for the simultaneous measurement of $U, \tau$ and $\rho$ in variable density flows. With the new instrumentation, developed for wall measurements, consisting of a wall aspirating probe and of a hot-wire/hotfilm interfering probes, experimental data on some properties at the wall has been obtained for the first time. In the flow studied here, characterized by a high level of density fluctuations, very consistent results have been obtained for all the measured quantities.

The present investigation shows that tangential injection of pure gases into a turbulent boundary layer of different density is characterized by a transition region which extends over a length of about 20-30 $e$ followed by a region of developed flow.

- It has been found that the flow is mainly governed by the initial velocity ratio $r$ and to a lesser extent by the density ratio $S$.

- The effect of density variations is small in the present experiment when $r$ is kept constant. Low values of $S$ increase the turbulent activity, especially in the transition region, which is consequently shorter in this case. This may be due to enhancement of large-scale coherent structures as shown by visualizations [9] and confirmed by the strong correlation between density and velocity fluctuation (Fig. 7).

- For $x / e>20-30$, the flow tends rapidly to an asymptotic state which is almost independent of the injected gas. Injection of helium, however, gives rise to a small increase of mean and turbulent velocities near the wall. Since the friction velocity $u_{\tau}$ obtained from the log law is also increased, the results scaled with wall variable are very close for the two cases of injection.

\section{REFERENCES}

[1]- LaRue J.C., Libby P.A., "Measurements in the turbulent boundary layer with slot injection of helium", Phys. Fluids, Vol. 20, n², (1977), pp.192-202.

[2]- LaRue J.C., Libby P.A., "Furthers results related to the turbulent boundary layer with slot injection of helium", Phys. Fluids, Vol. 23, n6, (1980), pp.1111-1118.

[3]- Cheng R.K, Ng T.T., "Some aspect of strongly heated turbulent boundary layer flow", Phys. Fluids, Vol. 25, n8, (1982), pp.1333-1341.

[4]- Sommer T.P., So R.M.C., Zhang H.S., "Heat Transfer Modeling and the assumption of zero wall temperature fluctuations", J. of Heat Transfer, Vol. 116, (1994), pp.855863.

[5]- Way J., Libby P.A., "Hot-wire probes for measuring velocity and concentration in helium-air gas mixtures", A.I.A.A.J., 3, n5, (1970), pp.976-978.

[6]- Purtell L.P., Klebanoff P.S., Buckley F.T., "Turbulent boundary layer at low Reynolds number", Phys. Fluids, Vol.24, n5, (1981), pp. 802-811.

[7]- Harion J.L., "Influence de différences de densité importantes sur les propriétés de transfert d'une couche limite turbulente", Thèse de doctorat INP Grenoble, (1994).

[8]- Harion J.L., Camano B.E., Favre-Marinet M., "Mesures de vitesse et de concentration par thermo - anémométrie dans des mélanges air - hélium", CRAS Paris, t320 Série IIb, (1995), pp.77-84.

[9]- Riva R., Binder G., Favre-Marinet M., Harion J-L., "Development of turbulent boundary layer with large density gradients", Exp. Thermal and Fluid Science, 9, (1995), pp.165-173.

[10]-Soudani A., "Application des réseaux de neurones aux mesures simultanées en écoulement turbulent", Thèse de doctorat INP Grenoble, (1996).

[11]- Soudani A., "Neural networks: A new method of calibration for the interference probe applied to measurements in air Helium turbulent boundary layer", Symposium on devices for flow measurement and analysis, USA, (1997).

[12]-Soudani A., Bessaih R., "Application of neural networks to measurements of simultaneous quantities in turbulent flows", International congress of fluid dynamics and propulsion, Cairo, Egypt, December 29-31 (1996).

[13]-Seban R.A., "Heat transfer and effectiveness for a turbulent boundary layer with tangential fluid injection", J. of Heat Transfer, Vol. 82, (1960), pp.303-312.

[14]-Harmett J.P., Birkebak R.C., Eckert E.R.G, "Velocity distributions, temperature distributions, effectiveness and heat transfer for air injected through a tangential slot into a turbulent boundary layer", J. of Heat Transfer, vol. 83, (1961), pp. 293-306.

[15]- Spalding D.B., "Prediction of adiabatic wall temperature in film-cooling systems", A.I.A.A.J., 3, n5 (1965), pp.965-967. 
